\title{
Surrogating Circuit Design Solutions with Robustness Metrics
}

\author{
Jin Sun ${ }^{\mathrm{a}, \mathrm{b}, *}$, Liang Xiao ${ }^{\mathrm{a}}$, Jiangshan Tian ${ }^{\mathrm{a}}, \mathrm{He}_{\mathrm{Zhou}}^{\mathrm{b}}$, Janet Roveda ${ }^{\mathrm{b}}$ \\ ${ }^{a}$ School of Computer Science and Engineering, Nanjing University of Science and Technology, No. 200 Xiaolingwei Street, Nanjing \\ 210094, China \\ ${ }^{b}$ Department of Electrical and Computer Engineering, The University of Arizona, 1230 E. Speedway Blvd., Tucson 85721, USA
}

\begin{abstract}
With the increase in device variability, the performance uncertainty poses a daunting challenge to analog $/ \mathrm{mixed}$-signal circuit design. This situation requires a robust design approach to add large margins to the circuit and system-level specification to ensure correct operation and the overall yield. In this paper, we propose a new robust design approach by using norm metric to quantify the robustness for both design parameter and performance uncertainty. In addition, we adopt a surrogating procedure to achieve robust design space and to reduce uncertainty in the performance space. The end result of the proposed method is a Pareto-surface that provides the designer with trade-offs between design robustness and performance uncertainty. One advantage of this new approach is the ability to take into account strong nonlinear relationship between performance and design parameters. Considering a set of highly nonlinear circuit performances, we demonstrate the effectiveness of this robust design framework on a fully CMOS operational amplifier circuit.
\end{abstract}

Keywords: Robust Design; Robustness Metric; ElasticR Method; Surrogates; Process Variations

\section{Introduction}

The continuous decrease of the CMOS device size leads to the increase in the process variations. With the increase in device variability, the performance uncertainty poses a daunting challenge to circuit design. For instance, a 5\% variation in transistor channel length may introduce up to $10 \mathrm{x}$ difference in leakage current and even causes functional failures in mixed-signal circuits [1 2] 3. Traditional approaches, such as timing analysis and yield estimation techniques [4 [5] 6], generally model circuit performance as a function in terms of design parameters. These parameterized models estimate performance fluctuation relying on the uncertainties in design parameters. It becomes apparent that the increasing device variability requires adding large margins to the circuit and system-level specification to ensure correct operation as well as the overall yield. Indeed, the ability to facilitate flexible designs is the key to improving circuit quality.

To ensure a safe, invulnerable design, it is of great importance to develop a design framework that is robust to parameter variations, and thus provides the designer with enough design margin. One popular methodology to improve circuit quality is robust design. This methodolo-

\footnotetext{
This work was supported by the National Science Foundation under grant nos. IIA-0926278, CCF-0915537 and CCF-0832282.

${ }^{*}$ Corresponding author.

Email addresses: sunj@njust.edu.cn (Jin Sun), xiaoliang@njust.edu.cn (Liang Xiao),

912106840410@njust.edu.cn (Jiangshan Tian),

hezhou@email.arizona.edu (He Zhou),

meilingw@email.arizona.edu (Janet Roveda)
}

gy focuses on simultaneously reducing parameter interval$\mathrm{s}$ and improving the fundamental functions of circuits or systems, and thus helps variable designs and concurrent engineering [7] 8. Over the past decades, many academic groups and companies are involved in traditional Taguchi method 9 10]11] 13. These efforts have had great impact on improving design quality and reducing design cost. However, many of them have reached the maximum potential due to their overly pessimistic nature.

Recently, several new attempts have been made for robust design. One category approaches use either design space exploration or norm concept to demonstrate design robustness. For example, 14 created an optimization methodology for generating a robust Pareto surface based on fast Monte-Carlo analysis. 15] proposed a norm-based metric to quantify design robustness in statistical timing analysis. This work predicts design parameter space in a backward mapping fashion. However, [15] does not provide a feasible optimization procedure for improving design robustness. On the other hand, a lot of efforts have been made to formulate robust design under parameter variations as a set-based deterministic problem. These method generally employs a uncertainty set to capture the variability in design parameters. The resulting set-based design problem can be further reduced to a deterministic one by constructing a solution that is robust to the variations within the uncertainty set. For example, the ellipsoidal uncertainty model [16 17] is widely used to characterize parameter variations as a deterministic ellipsoidal set. 18] further proposes a conic representation of uncertainty set, of which the size are associated with not only the nominal 
design point, but also the specified yield requirement. All these set-based approaches assume that an explicit and accurate model of circuit performance has been established before solving the optimization problem.

In many scenarios, the objective and constraint functions in robust design framework are implicit and expensive, due to the increasing complexity of integrated circuits. To tackle this problems, surrogate models are widely researched to approximate circuit performance based on cost-effective model templates. Surrogate models are built on sampling data obtained from SPICE-like simulations. Response surface model (RSM) is a traditional method widely used in circuit performance modeling. However, RSM is incapable of capturing the strong nonlinearity of circuit performance upon parameter variations. Moreover, RSM requires a huge number of samples when constructing the surface model. In recent years, more researches have been conducted to develop other power surrogate models, including Kriging, rational and radial basis functions [19 20 21. For example, 19 proposes using Kriging model combined with Latin Hypercube Sampling to build a reasonable surrogate model of circuit performance.

Above existing approaches model circuit performance as linear or piece-wise linear functions in terms of design parameters. Therefore when evaluating highly nonlinear circuit performances, such as gain-bandwidth, phase margin, etc., these approaches fail to capture the nonlinear relationships between performances and design parameters, and tend to predict non-robust design solutions. Despite its growing importance in today's circuits and applications, achieving robustness with highly nonlinear circuit performances has not been addressed enough by existing researches. Such cases observe major performance shifts with very insignificant parameter variations, and thus demand limiting performance uncertainty while allow maximum robustness for design parameters.

In this paper, we propose a new robust design framework that includes both forward mapping and backward mapping procedures. We consider a set of circuit performances with nonlinear/implicit function expressions of design parameter. Starting with user-defined performance constraints, we first apply backward mapping [22] 23$] 24$ to identify feasible regions for design parameters. Then we propose an ElasticR method to measure the norm distance from the nominal design solution to any design points that will cause performance violation. The minimum distance thus generated demonstrates the robustness for design parameters. In addition, a forward mapping procedure [25] [26] is applied to measure the robustness for performance fluctuations induced by parameter variations. Similarly, we measure the maximum norm distance between nominal performance point and performance fluctuations. After quantifying the robustness metrics in both parameter space and performance space, we then identify optimal nominal design parameters that have the maximum robustness while minimizing performance fluctuation. This optimization procedure is implemented by employing sur- rogating techniques 27 28 to efficiently search for optimal design solutions. The contributions of this paper includes: (1) proposing a two-way mapping procedure to ensure robustness for design parameters and performance variations, (2) developing an ElasticR method of robustness quantification to estimate design parameter boundaries as well as their associated performance boundaries, and (3) providing surrogate-based optimization procedure to iteratively search optimal design solutions among a group of candidates. The proposed methodology was verified on an analog operational amplifier circuit. Experimental results show that this two-way mapping approach has achieved up to $38 \%$ robustness improvement compared with oneway mapping procedures.

The rest of this paper is organized as follows. Section 2 defines two robustness metrics both in parameter space and performance space based on norm distance. Section 3 details the surrogate-based robust design framework for robustness metrics. Experimental results are presented in Section 4. Finally, Section 5 concludes this paper.

\section{Quantifying Robustness for Parameter and Per- formance}

This section introduces two robustness metrics in both parameter space and performance space. An ElasticR method is developed for the quantification of both robustness metrics.

\subsection{Parameter Robustness}

Consider a group of design parameters $L_{\text {eff } 1}, W_{1}, T_{\text {ox } 1}, L_{\text {eff } 2}, W_{2}, T_{\text {ox } 2}, \cdots$, representing effective channel length, width, oxide thickness for each device. To simplify the notation, we use vector $X=\left(x_{1}, x_{2} \cdots, x_{n}\right)$ to represent all design parameters, with parameter variations $\Delta X$ around their nominal values $X^{0}$. Denoted by $Y=\left(y_{1}, y_{2}, \cdots, y_{m}\right)=f(X)$, the corresponding circuit performances may include gain-bandwidth, phase margin, slew rate and other performance metrics. Likewise, $Y^{0}$ and $\Delta Y$ represent the nominal performance metrics and the corresponding perturbations.

In robust design, there exist performance limits, $Y^{L}=$ $\left(y_{1}^{L}, y_{2}^{L}, \cdots, y_{m}^{L}\right)$ and $Y^{U}=\left(y_{1}^{U}, y_{2}^{U}, \cdots, y_{m}^{U}\right)$, such that the fluctuations in circuit performance due to parameter variations satisfy:

$$
y_{j}^{L} \leq \Delta y_{j}=y_{j}-y_{j}^{0} \leq y_{j}^{U}, j=1, \cdots, m
$$

where $y_{j}^{L}$ and $y_{j}^{U}$ indicate the maximum acceptable fluctuations for $j$-th circuit performance. On the other hand, parameter variations also have lower bound $X^{L}$ and upper bound $X^{U}$ that come from design rule requirements. In general, the values of $X^{L}$ and $X^{U}$ may vary depending on the location of nominal design in parameter space.

A good design should satisfy that design parameters will lead to performances bounded by the performance specifications. We define feasible region as a set in parameter 
space consisting of all design candidates that produce performance metrics satisfying the performance constraints. Or in other words, the performance constraints map backward into parameter space and forms a parameter feasible region (FR). Note that design parameters themselves are limited by their variation bounds $X^{L}$ and $X^{U}$. In a rigorous manner, for any design candidate $X^{0}$ whose robustness to be evaluated, considering parameter variations and performance constraints, there will be a set of acceptable variations in parameter space, such that the performance fluctuations propagated from this set will not violate the performance constraints:

$$
\mathrm{FR}=\left\{\Delta X \mid Y^{L} \leq \Delta Y \leq Y^{U}, X^{L} \leq \Delta X \leq X^{U}\right\}
$$

where $Y^{L}$ and $Y^{U}$ represent the performance specification$\mathrm{s}$, and $\Delta Y$ denote the performance perturbations due to parameter variations: $\Delta Y=f\left(X^{0}+\Delta X\right)-f\left(X^{0}\right)$. The region defined in (2) includes all feasible parameter variations that satisfy performance constraints, and therefore is named as parameter feasible region (FR). Figure 1 shows an example of a FR in 2-dimensional parameter space. We observe that FR is specified by both parameter variation bounds and performance specifications. A point inside FR (Point A) or on the boundary of FR (Point B) represents a feasible variation value that satisfies the performance constraints. On the other hand, a point outside FR represents a perturbation value that violates the performance constraints (Point $\mathrm{C}$ ), or exceeds parameter variation range (Point D).

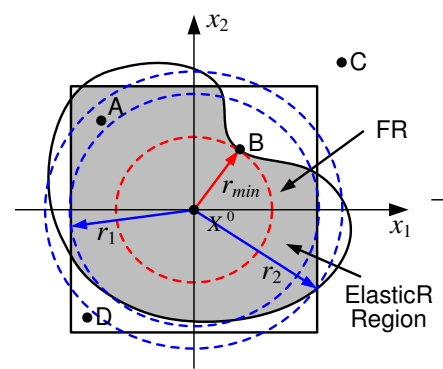

(a) Design 1: greater $r_{\text {min }}$, more robustness

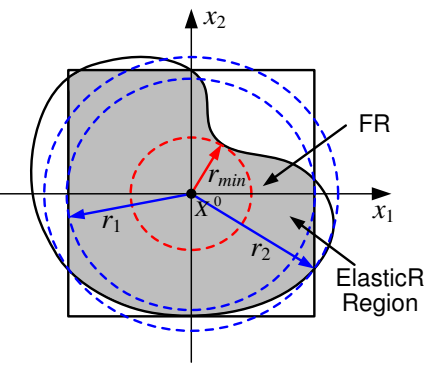

(b) Design 2: smaller $r_{\text {min }}$, less robustness
Figure 1: Parameter Feasible Region (FR)

We suggest using a distance-based metric to quantify the robustness for design parameters within the feasible region. Norm distance is beneficial to describing the large dimensionality of robust design by presenting a simple quantification measure [15]. For any point $X=$ $\left(x_{1}, x_{2} \cdots, x_{n}\right)$ in $\mathrm{n}$-dimensional space, the distance from the origin to $X$ can be described used norm metrics, $\|\cdot\|_{q},(q=1,2, \cdots, \infty)$, as follows:

$$
L_{q} \text {-norm }=\left\{\begin{array}{l}
\|X\|_{1}=\sum_{i=1}^{n}\left|x_{i}\right|, q=1 \\
\|X\|_{2}=\left(\sum_{i=1}^{n} x_{i}^{2}\right)^{\frac{1}{2}}, q=2 \\
\|X\|_{\infty}=\max _{i=1, \cdots, n}\left|x_{i}\right|, q=\infty
\end{array}\right.
$$

As suggested in 15, we use $L_{2}$-norm norm distance to quantify the robustness in parameter space. Specifically, we define the robustness metric as the minimum distance from the nominal point (i.e. the center point of FR) to any design point where performance constraint violation occurs, or equivalently the minimum distance from the nominal point to the closest boundary of FR. As shown in Figure 1, the minimum distance $r_{\text {min }}$ in fact reflects the smallest deviation from the nominal point that would break this design. The nominal design is expected to be optimized such that this distance is maximized, in the expectation of maximizing yield. For example, we observe two different designs whose FR's are illustrated in Figure 1(a) and Figure 1(b) respectively: design 1 has greater $r_{\text {min }}$ distance than design 2 , indicating that design 1 allows more headroom for parameter variations before touching the boundary of FR. Therefore we conclude that design 1 is more robust than design 2 . In this sense, such distance $r_{\text {min }}$ is defined as a measure of robustness in parameter space: the more distant the nominal point is from the FR boundary, the higher is the expected yield. It is worth emphasizing that when quantifying robustness, all parameter variations have been standardized into the interval $[-1,1]$, in order to eliminate the effect of units.

In what follows, by employing the concept of a Second Order Cone (SOC) we introduce the ElasticR method for norm-based robustness quantification. Mathematically, a second order cone of dimension $k$ is defined as [29]:

$$
\left\{\left[\begin{array}{c}
U \\
t
\end{array}\right] \mid U \in R^{k-1}, t \in R,\|U\| \leq t\right\}
$$

where $U$ is a vector of dimension $k-1$. Following the definition in (4) we define a SOC representation in design parameter space, by introducing an auxiliary variable $r$ :

$$
\left\{(X, r) \mid\left\|X-X^{0}\right\|_{2} \leq r, r \geq 0\right\}
$$

where $\|\cdot\|_{2}$ denotes the 2-norm distance from the center point $X^{0}$. The SOC representation in (5) is with $n+1$ dimensions because of the introduced variable $r$. In parameter space such $\mathrm{SOC}$ in fact denotes a hyper-sphere with elastic radius $r$. By this token we name this quantification approach as ElasticR method. Referring to the example in Figure 1, the SOC reduces to a circle in 2dimensional space, and radius $r$ restricts how far parameter variations can perturb from the nominal point. This SOC representation allows continuously changing radius $r$ to capture all the boundary points of FR, and further determine the minimum distance of the closest boundary point. As shown in Figure 1, the continuously varying $r$ variable defines a ElasticR region in parameter space. Any specified value of $r$ provides a particular boundary point distance, e.g. $r_{1}$ and $r_{2}$. By varying the elastic variable $r$ we can determine the minimum radius with which the ElasticR region touches the boundary of FR at the closest point (e.g. Point B in Figure 1(a)). In this scenario, the minimum radius of ElasticR region, denoted by $r_{\text {min }}$, is 
identified as the robustness metric for design parameters, and is denoted by $R_{x}$. Note that robustness $R_{x}$ depends on where the nominal point is located.

Having defined parameter robustness by ElasticR method, the most challenging problem for designers is how to map the performance constraints backward into parameter space and further evaluate the size of FR. In general for many nonlinear circuit performances, however, the mathematical formulation of the FR's shape may not unknown or implicit 22]. In many scenarios, the performance function may even be a black box. As a consequence, an analytical calculation of the minimum distance within FR is not feasible.

In order to address above-mentioned issue, we provides an indirect way of computing $R_{x}$, i.e. the minimum radius of ElasticR region. This is done by establishing an optimization problem in order to avoid analytical mapping from performance constraints [22]. By definition, the minimum radius of ElasticR region is the (2-norm) distance from the nominal point to a closest point on FR boundary. Following two facts: (1) this point has minimum distance from the nominal point; and (2) this point is on the boundary of FR where performance violation occurs, we can determine $R_{x}$ by solving the following optimization problem:

$$
\begin{array}{cc}
\text { minimize } & r \\
\text { subject to } & r=\left\|X-X^{0}\right\|_{2} \\
& \min _{j=1, \cdots, m}\left\{\Delta y_{j}-y_{j}^{B}\right\}=0 \\
\Delta y_{j}=f_{j}(X)-f_{j}\left(X^{0}\right) \\
X^{L} \leq X-X^{0} \leq X^{U}
\end{array}
$$

where $y_{j}^{B}$ denotes the $j$-th performance specification $y_{j}^{L}$ or $y_{j}^{U}$. The first constraint in 6 follows SOC definition, the second constraint indicates that the point to be determined is located on the boundary of FR, and robustness $R_{x}$ is measured as the distance of this particular point from the nominal point.

\subsection{Performance Robustness}

As mentioned previously, this robust design framework is a two-way mapping procedure, accounting for not only parameter robustness in design space, but also performance robustness in objective space. Performing optimization only in parameter space may cause large fluctuation in performance space. In a similar manner, we define a distance-based robustness metric to measure the maximum performance fluctuation, an the ElasticR method is applied to quantify the robustness metric of performance fluctuations.

For a design candidate with parameter variations, the set of all possible perturbations it maps forward into performance space, is defined as a performance variation region (PVR):

$$
\mathrm{PVR}=\left\{\Delta Y=f\left(X^{0}+\Delta X\right)-f\left(X^{0}\right) \mid X^{L} \leq \Delta X \leq X^{U}\right\}
$$

Figure 2 shows an example of PVR in 2-dimensional performance space. The PVR includes all possible outcomes of performance fluctuation propagated from parameter variations. For each performance metric the performance

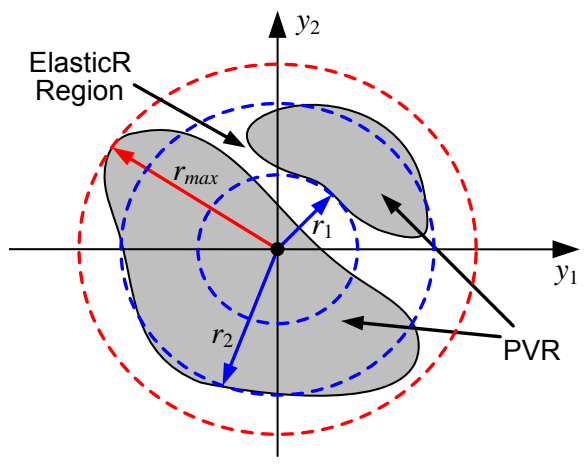

Figure 2: Performance Variation Region (PVR)

perturbation has been normalized to its nominal value. We use the maximum distance from the nominal point to any fluctuation point in PVR, i.e. the distance of the furthest point on the boundary of PVR from the nominal point, to measure the robustness for performance fluctuations.

Similarly as in quantifying parameter robustness, in performance space we introduce the ElasticR region with elastic radius $r$. The ElasticR method employs a set of different radiuses $\left(r_{1}, r_{2}, \cdots\right)$ to capture all fluctuation points on the PVR boundary and quantify their perturbation distances, as shown in Figure 2, The maximum radius $r_{\max }$ will be identified when the ElasticR region reaches the most distant point on PVR boundary. The maximum radius of ElasticR region is then used to measure the robustness metric for performance fluctuations. Since PVR is mapped forward from parameter variations, the maximum distance $r_{\max }$ is also dependent on the location of nominal design. A good design is expected to minimize the distance of the furthest fluctuation point. Further distance indicates wider range of performance fluctuations, and in turn worse yield.

Directly calculating the maximum radius of ElasticR region for every possible design value will in general be intractable because PVR must be covered finely enough. In an even worse case, the performance function may be a black box. Likewise, the distance $r_{\max }$ can be indirectly calculated using an optimization procedure:

$$
\begin{array}{cc}
\text { maximize } & r \\
\text { subject to } & r=\|\Delta Y\|_{2} \\
& \Delta Y=Y(X)-Y\left(X^{0}\right) \\
& X^{L} \leq X-X^{0} \leq X^{U}
\end{array}
$$

By solving above optimization problem we obtain the furthest fluctuation distance $r_{\max }$ on the PVR boundary. The greater this distance is, the less robust is this design, since greater $r_{\max }$ implies higher possibility of performance fluctuation exceeding the specification values. Therefore 
performance robustness $R_{f}$ is defined as:

$$
R_{f}=\left\|Y^{B}\right\|_{2}-r_{\max }
$$

where $Y^{B}$ denotes performance bounds $Y^{L}$ or $Y^{U}$, and $\left\|Y^{B}\right\|_{2}$ indicates the maximum acceptable distance for performance fluctuations. Robustness $R_{f}$ in 9 determines the headroom from performance fluctuation to its maximum acceptable range.

To summarize, two distance-based metrics are introduced to quantify the robustness metrics both for design parameters and performance fluctuation. Starting with PBR, a feasible region in parameter space FR will be determined by backward mapping. By using the ElasticR method, the minimum distance of FR boundary points to the nominal point is identified as the parameter robustness for this design. Moreover, the FR maps forward into the performance space and forms the PVR with an ElasticR region evaluating the greatest performance perturbation. The robustness metric for performance fluctuation will be measured as the size of such ElasticR region. It is worth emphasizing that, though for illustrative purpose, the figures in this section use two performance constraints to interpret the robustness concepts, the robustness metrics defined in (6) and (8) can be applied to scenarios with more than two performance functions.

\section{Surrogating Circuit Design with Robustness Metrics}

The goal of this proposed design framework is to determine the best nominal settings of design parameters, in the presence of parameter variations and performance specifications. The nominal design should be optimally located such that the robustness for design parameters is maximized, at the same time the performance fluctuation can be manipulated to an acceptable extent. The optimal solutions provide the designer with trade-off information between design robustness and performance uncertainty.

\subsection{Robust Optimization Model}

The two robustness metrics, $R_{x}$ and $R_{f}$, both depend on the location of nominal design with implicit function relationships (refer to (6) and (8)). Both robustness metrics are optimized simultaneously. The robust design problem is formulated as a multi-objective optimization problem:

$$
\begin{array}{cc}
\text { maximize } & R_{x}(X) \\
\text { maximize } & R_{f}(X) \\
\text { subject to } & Y^{L} \leq \Delta Y \leq Y^{U} \\
& X^{L} \leq \Delta X \leq X^{U}
\end{array}
$$

where $\Delta Y=f(X+\Delta X)-f(X)$. We solve this problem in a Pareto sense, thus designers are able to choose the solution(s) according to their requirements. AWS (Adaptive Weighted Sum) method [30] is an effective way to generate a set of uniformly-distributed Pareto solutions. Though there are various algorithms for multi-objective optimization (e.g. conventional weighted sum method 31 and normal boundary insertion method 32), the advantage of AWS method is its ability to provide a well-distributed Pareto front even in non-convex design regions. By assigning a weighting factor $\alpha$ for two objectives, the optimization problem in 10 can be translated into a singleobjective problem:

$$
\begin{array}{cc}
\operatorname{maximize} & \alpha R_{x}(X)+(1-\alpha) R_{f}(X) \\
\text { subject to } & Y^{L} \leq \Delta Y \leq Y^{U} \\
& X^{L} \leq \Delta X \leq X^{U}
\end{array}
$$

Then by adaptively updating the weighting factor, we can obtain a set of solutions evenly distributed on the Pareto surface. However, in each step with fixed weighting factor, it is necessary to develop an efficient algorithm to solve the complicated optimization problem in (11). During each iteration procedure, the backward mapping and forward mapping are performed to evaluate the two robustness metrics in the objective function. Note that the robustness metrics are determined by solving sub-optimization procedures (6) and (8), and therefore are implicit functions in terms of design parameters. In this situation, the surrogate modeling would be necessary to make this problem tractable.

\subsection{Robust Design Using Surrogates}

As described in Section 2, both parameter robustness and performance robustness are obtained by solving optimization problems (refer to (6) and (8)), hence the objective function in (11) is implicit and expensive to solve. We take advantage of SMF (Surrogate Management Framework) to overcome this difficulty. SMF [27] 28] is a rigorous optimization algorithm for some design problems in which the functions are expensive black boxes, or accurate approximations are problematic. Different from existing approximate- or fitting-based methods, SMF method solves such optimization problems by generating a sequence of cost-effective approximations to the objective function and efficiently managing these approximation$\mathrm{s}$ as surrogates for optimization. In addition, fittingbased methods require large number of sampling points and may cause serious over-fitting issues in case of a highdimensional design space. The polling procedure in SMF method guarantees that the surrogate model converges to the true performance function without over-fitting concerns 28]. Furthermore, SMF method does not require any assumptions of surrogates and therefore facilitates the solver when solving the formulated problem 11 .

A preliminary issue in this surrogate-based framework is how to build surrogates. We start with running circuit simulations on certain design candidates, and quantifying the robustness metrics for these selected designs. We then construct the surface response models for the objective function based on the experimental designs, and further use those approximations to build optimal surrogates. 
More details can be referred to 33 34. In what follows, we assume that a family of surrogating functions has been specified that an initial surrogate has been constructed.

The procedure of surrogate-based optimization is a set of strategies for using surrogates in both the search and poll steps 28. In each step the surrogating function has been updated until the surrogating solution is sufficiently close to the real optimal solution. Suppose that $s_{0}$ is the initial surrogate for the objective function of robustness, and $X_{0}^{*}$ the initial solution point. We decompose the feasible design space (within FR) into a mesh grid $M_{0}$ with the same dimensions. Also we let $P_{0} \subset M_{0}$ contain $X_{0}^{*}$ and any points adjacent to $X_{0}^{*}$. As the algorithm generates $X_{k}^{*} \in M_{k}$ in each iteration step, let $P_{k} \subset M_{k}$ be defined by the same token. For $k=0,1,2, \cdots$ the algorithm performs the following steps:

Step 1: Search on $s_{k}$, which is the $k$-th surrogate of objective function, to find a better point $X_{k+1}^{*} \in M_{k}$.

Step 2: If such $X_{k+1}^{*}$ in Step 1 is identified, which means that $X_{k+1}^{*}$ achieves improvement to $X_{k}^{*}$, then set $M_{k+1}=$ $M_{k}$ and update the surrogate with the newly extracted point $X_{k+1}^{*}$; else go to Step 3 .

Step 3: If the search procedure in Step 2 fails, poll $s_{k}$ in the set $P_{k}$ (including $X_{k}^{*}$ and all its adjacent points). If a better point is explored, then set $M_{k+1}=M_{k}$ and update the surrogate; else go to Step 4.

Step 4: If $X_{k}^{*}$ is already the optimal point in $P_{k}$, then set $X_{k+1}^{*}=X_{k}^{*}$, refine the mesh grid $M_{k+1}=M_{k} / 4$, and update the surrogate; else return to Step 1.

For the robust design problem in (11), any point on the current mesh that achieves better robustness can be used in the next iteration. The exploration of such improving points is the key basis to guarantee the convergence to the optimal solution 35. Surrogate-base algorithm iteratively evaluates and recalibrates the objective function at a predetermined subset of current mesh, in order to predict points at which we expect to realize improvement. The set $P_{k}$ contains the list of potential candidates, and we choose those most promising candidates. It is worth emphasizing that the reuse of the surrogate or not depends on whether the designer is satisfied with the accuracy of surrogate model. If the current surrogate does not predic$\mathrm{t}$ any better point, we then have to refine the mesh and poll again. More specifically, if the current iteration is sufficiently near a minimizer of the true objective function, then the current mesh must be refined in order for optimization to progress. The surrogate model can be reused if additional points are selected for surrogating, and the polling procedure thus will continue.

Algorithm 1 describes the algorithmic flow of surrogatebased robustness optimization. The algorithm acts as a speculative search procedure in design parameter space. We continue to iterate this search procedure (lines 6 10 ) as long as it finds an improved design solution, and we use the objective function values of robustness to recalibrate the surrogates (line 10 at each iteration. When the search procedure fails, a local polling step is performed

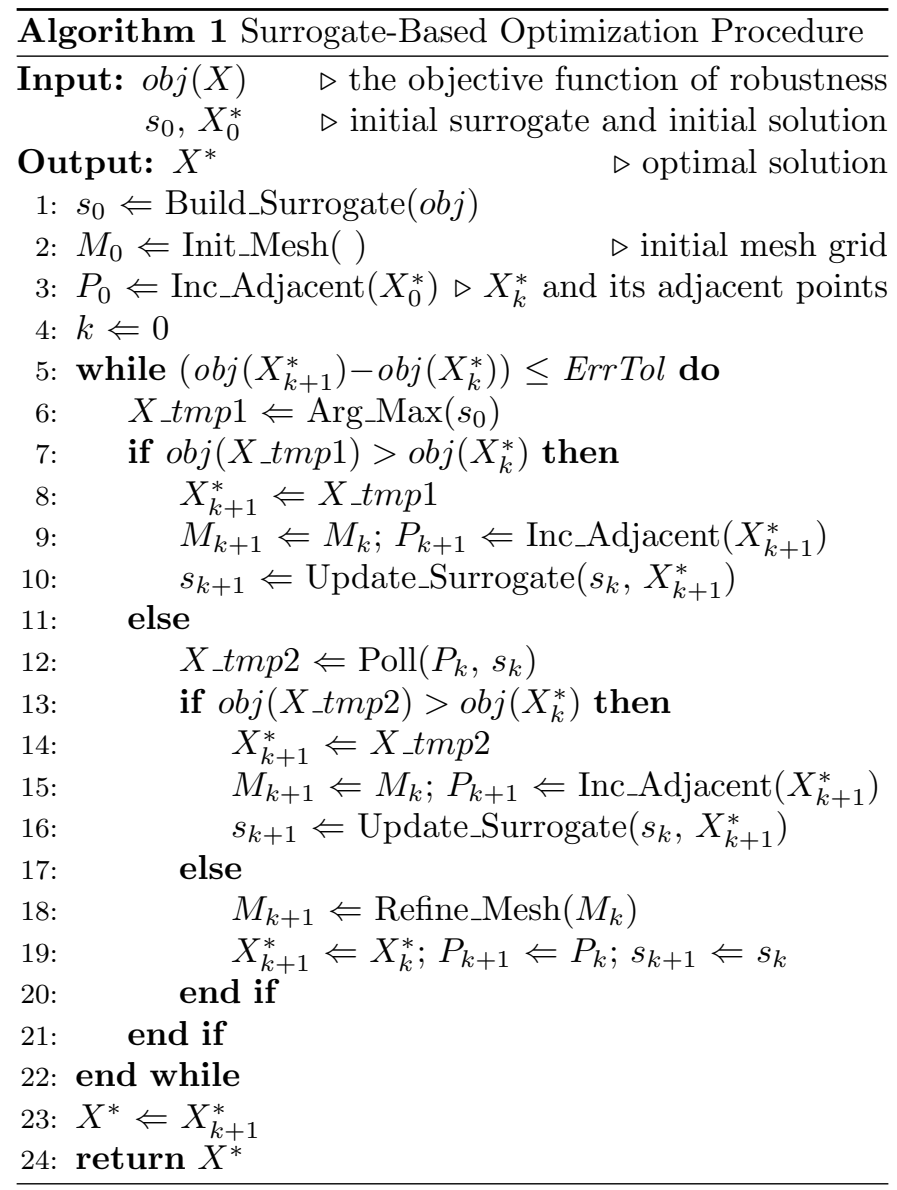

on the set of adjacent points (line 12), in order to determine if we need to refine the mesh before we recalibrate the surrogates. It is the polling step that guarantees the convergence of the algorithm (lines 12 16). If mesh refining is necessary, which means that the polling step could not exploit any improving point, the mesh is reorganized with higher resolution, and new surrogates will be further evaluated and updated accordingly (lines 18,19 .

As mentioned above, both robustness metrics are optimized simultaneously and we solve the robust design problem is a Pareto sense. The end result is a set of Pareto solutions providing trade-off information between parameter robustness and performance variation. The general flow of the proposed robust design framework is summarized in Figure 3. In the presence of parameter variations and performance constraints, backward mapping and forward mapping are employed to quantify robustness metrics for parameter variations and performance fluctuation. By introducing a weighting factor $\alpha$ we formulate the robust design problem into a single-objective optimization program 11). Algorithm 1 facilitates exploring an optimal design setting for any fixed $\alpha$ value. In surrogate-based optimization procedure, circuit simulation and function approximation are conducted to build surrogates for the robustness objective function. Then based on the constructed surrogates, an optimal design solution will be identified by following the computational flow described in Algorithm 


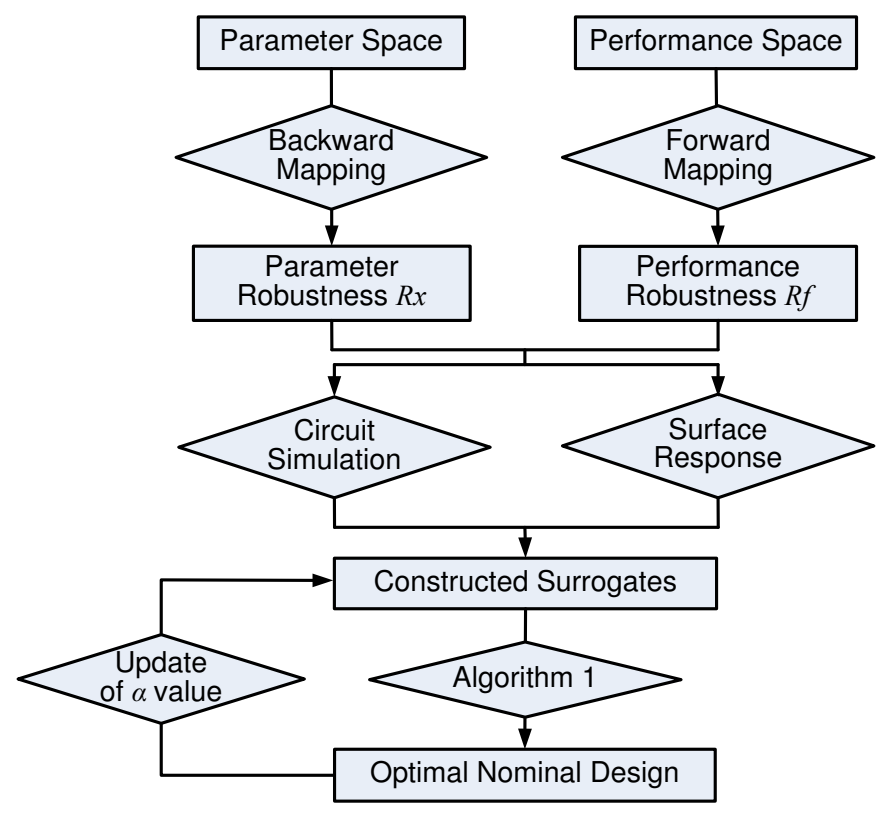

Figure 3: Flowchart of the robust design framework

1. Only few number of circuit simulations are required to build the surrogate functions, but the complicated optimization problem can be solved efficiently. By adaptively updating the weighting factor $\alpha$, a well-distributed Pareto surface will be generated.

\section{Experimental Results}

The proposed robust optimization framework was implemented by MATLAB programming and HSPICE simulations. All simulations and experiments were performed on a quad-core 3.4-GHz machine with 16-GB memory. A fully CMOS operational amplifier (opamp) circuit using 90nm technology was selected for verification. The schematic of this opamp circuit is illustrated in Figure 4. In robust-

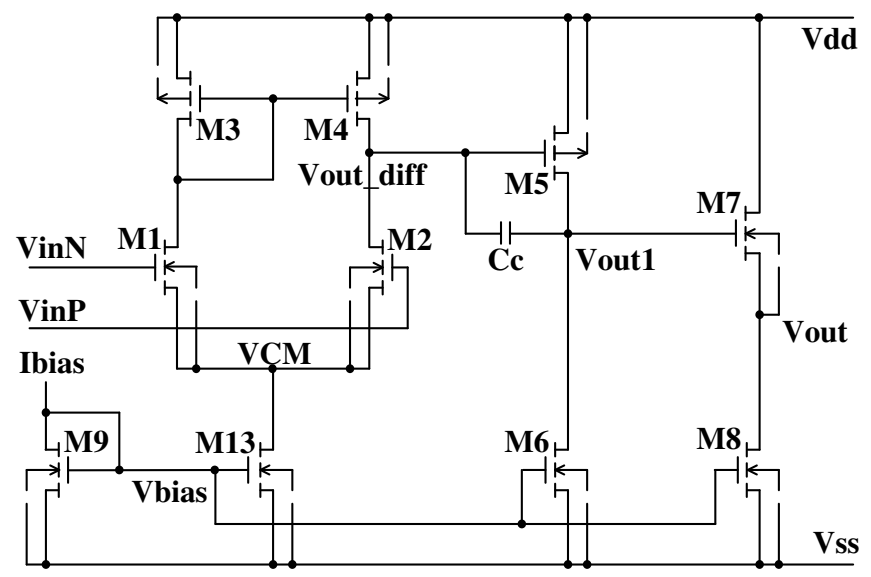

Figure 4: The schematic of the opamp circuit

ness analysis we mainly consider MOSFET effective channel lengths ( $L_{\text {eff's }}$ ) and widths $(W$ 's) as design parameters.
All $L_{\text {eff }}$ and $W$ parameters are assigned $30 \%$ process variations around their nominal values. Note that the variation range of each parameter has been normalized to the unit interval $[-1,1]$. In performance space we evaluate the perturbation in gain-bandwidth (denoted as $y_{1}$ ), phase margin $\left(y_{2}\right)$ and slew rate $\left(y_{3}\right)$. The performance specifications $\left(y_{i}^{L}\right.$ and $\left.y_{i}^{U}\right)$ are initially set as $10 \%$ of the nominal performance value: a design candidate is considered feasible only if all performance fluctuations propagated from its associated variations are within $10 \%$ of the nominal value of performance metric.

To verify the correctness of robust design solutions, we first run Monte-Carlo simulations and generate 2,000 sample designs. For each sample of design candidate, we follow the quantification procedure presented in Section 2 to evaluate its parameter robustness $\left(R_{x}\right)$ and performance robustness $\left(R_{f}\right)$. A design with smaller robustness metric is more prone to failure. Figure 5 shows the robustness distributions for this opamp circuit. The upper two figures

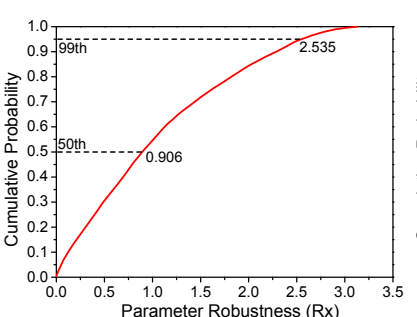

(a) $R_{x}$ cumulative probability

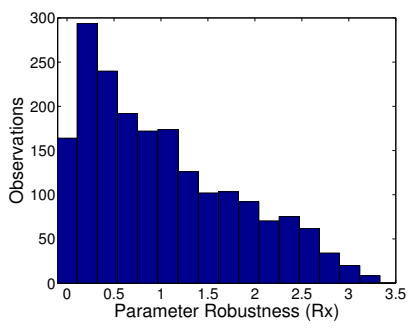

(c) $R_{x}$ distribution

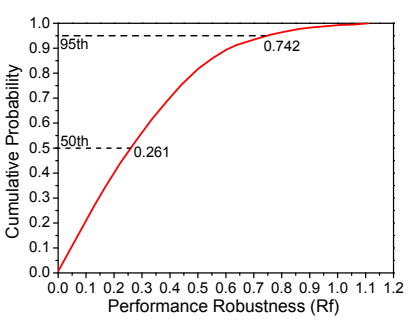

(b) $R_{f}$ cumulative probability

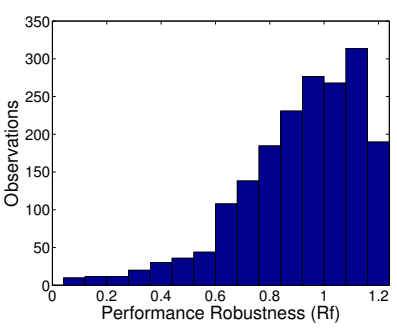

(d) $R_{f}$ distribution
Figure 5: Distributions of Robustness metrics

$5(\mathrm{a})$ and 5(b) present the cumulative distribution functions (CDFs) for $R_{x}$ and $R_{f}$ respectively. The x-axis denotes a specific value of robustness metric $R_{0}$, and the y-axis gives the cumulative probability for this particular robustness metric. CDF is useful in verifying the performance yield since it directly indicates the cumulative probability information. In general it is useful to set a robustness threshold such that all design candidates with robustness less than this threshold value would be considered vulnerable to parameter variability, and therefore need to be fixed for purpose of robustness improvement. The lower two figures 5(c) and 5(d) illustrate the histograms of $R_{x}$ and $R_{f}$ distributions generated by Monte-Carlo simulations.

As claimed before, this robust design framework is a two-way mapping procedure: the robustness for design 
parameters and robustness for performance perturbations are both regarded as design objectives and optimized simultaneously. The end result is a set of design solutions in Pareto-optimal sense, providing the designer with the flexibility to choose the most appropriate solution(s) according to specific design requirements. Figure 6 presents the robust design results in $R_{x}-R_{f}$ space. Each blue point

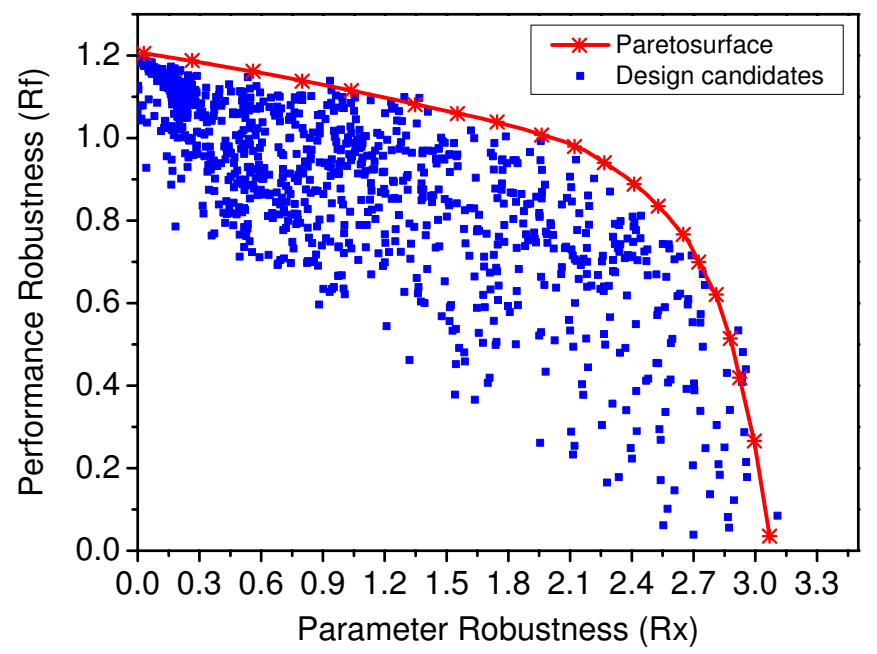

Figure 6: Pareto surface of robustness metrics

denotes the quantified robustness metrics $\left(R_{x}\right.$ and $\left.R_{f}\right)$ for a particular sample design candidate. The red curve represents the Pareto surface predicted by the proposed flow. Since each sample of feasible design candidate corresponds to a pair of robustness metrics $\left(R_{x}, R_{f}\right)$ in robustness space, the complete robustness space (formed by all feasible design candidates) can be approximated by Monte-Carlo results. According to the definition of Pareto optimality, all Pareto solutions are supposed to be distributed on the boundary of robustness space. The results in Figure 6 show that the predicted Pareto surface matches well with Monte-Carlo results. Depending on specific robustness requirements, the designer may choose a specific solution on the generated Pareto surface as the nominal design that yields the Pareto-optimal robustness metric. If a better robustness metric is expected in parameter/performance space, a higher weight should be assigned to the parameter/performance robustness function.

To demonstrate the accuracy of Pareto solutions obtained by using surrogate models, we further conduct robustness optimization without using surrogate model and compare the results with surrogate-based results. Relying on the Monte-Carlo sampling data, we investigate each sample of feasible design and its corresponding robustness metrics. Instead of the surrogating procedure, we use a fitting procedure to approximate the dependency of robustness upon design parameters. A part of the resultant Pareto solutions are listed in Table 1 and compared with the results obtained by using surrogate modeling. For different $\alpha$ values, surrogate-based method has achieved very similar results compared with results obtained with- out surrogate modelings. A minor difference (less than $5 \%$ ) can be observed from Table 1 .

Table 1: Robustness metrics with surrogate modeling and without surrogate modeling

\begin{tabular}{|c|c|c|c|c|c|}
\hline Weighting & \multicolumn{2}{|c|}{ W/ SM } & \multicolumn{2}{c|}{ W/O SM } & Max \\
\cline { 2 - 5 } Factor $(\alpha)$ & $R_{x}$ & $R_{f}$ & $R_{x}$ & $R_{f}$ & Diff. \\
\hline$\alpha=0.1$ & 0.2963 & 1.1924 & 0.2818 & 1.1484 & $4.90 \%$ \\
\hline$\alpha=0.2$ & 0.3907 & 1.1534 & 0.3756 & 1.1144 & $3.87 \%$ \\
\hline$\alpha=0.4$ & 1.5457 & 1.0647 & 1.5017 & 1.0160 & $4.58 \%$ \\
\hline$\alpha=0.6$ & 2.2542 & 0.9435 & 2.1894 & 0.9061 & $3.96 \%$ \\
\hline$\alpha=0.7$ & 2.5161 & 0.8375 & 2.4370 & 0.8050 & $3.87 \%$ \\
\hline$\alpha=0.8$ & 2.7179 & 0.7033 & 2.5928 & 0.6696 & $4.79 \%$ \\
\hline$\alpha=0.9$ & 2.9068 & 0.5172 & 2.8157 & 0.5006 & $3.21 \%$ \\
\hline
\end{tabular}

To emphasize the importance of using two-way mapping procedure, we choose several Pareto solutions and compare them with the results obtained by one-way mapping method. Table 2 shows the robustness optimization results by using two-way mapping procedure and one-way forward/backward mapping procedure, respectively. In one-way mapping procedure the optimization is performed solely in design space or performance space, without the multi-optimization procedure of both robustness metrics. A robustness improvement ranging from $8 \%$ to $38 \%$ achieved by using the proposed method can be observed.

Table 2: Robustness metrics by using two-way mapping and one-way mappings

\begin{tabular}{|c|c|c|c|c|c|c|}
\hline \multirow{2}{*}{$\begin{array}{c}\text { Pareto } \\
\text { Solns }\end{array}$} & \multicolumn{2}{|c|}{ 2-Way } & \multicolumn{2}{c|}{ Forward } & \multicolumn{2}{c|}{ Backward } \\
\cline { 2 - 7 } & $R_{x}$ & $R_{f}$ & $R_{x}$ & Improv. & $R_{f}$ & Improv. \\
\hline Soln 9 & 1.9664 & 1.0098 & 1.8213 & $7.90 \%$ & 0.7785 & $29.71 \%$ \\
\hline Soln 10 & 2.1217 & 0.9716 & 1.8213 & $16.49 \%$ & 0.7785 & $24.80 \%$ \\
\hline Soln 11 & 2.2542 & 0.9435 & 1.8213 & $23.77 \%$ & 0.7785 & $21.19 \%$ \\
\hline Soln 12 & 2.4025 & 0.9209 & 1.8213 & $31.91 \%$ & 0.7785 & $18.29 \%$ \\
\hline Soln 13 & 2.5131 & 0.8980 & 1.8213 & $37.98 \%$ & 0.7785 & $15.35 \%$ \\
\hline
\end{tabular}

We further perform another set of experiments to further demonstrate the effectiveness of the proposed methodology. We select different performance specification values and present the corresponding robust design solutions in Figure 7. The specification values for performance perturbations are set at $8 \%, 13 \%$ and $15 \%$ of their nominal values respectively. In all cases our design framework is capable of predicting a well-distributed set of Pareto solutions of robustness metrics.

At last, the runtime statistics by using the proposed twoway mapping approach and one-way mapping approaches are summarized in Table 3 Different from backward mapping that optimizes robustness metric solely in design space without looking into performance variations, and forward mapping that optimizes robustness metric solely by characterizing fluctuations in performance space, the two-way mapping method optimizes both parameter robustness and performance robustness simultaneously, and therefore leads to higher computational cost. However, with a set of well-distributed Pareto solutions, the pro- 


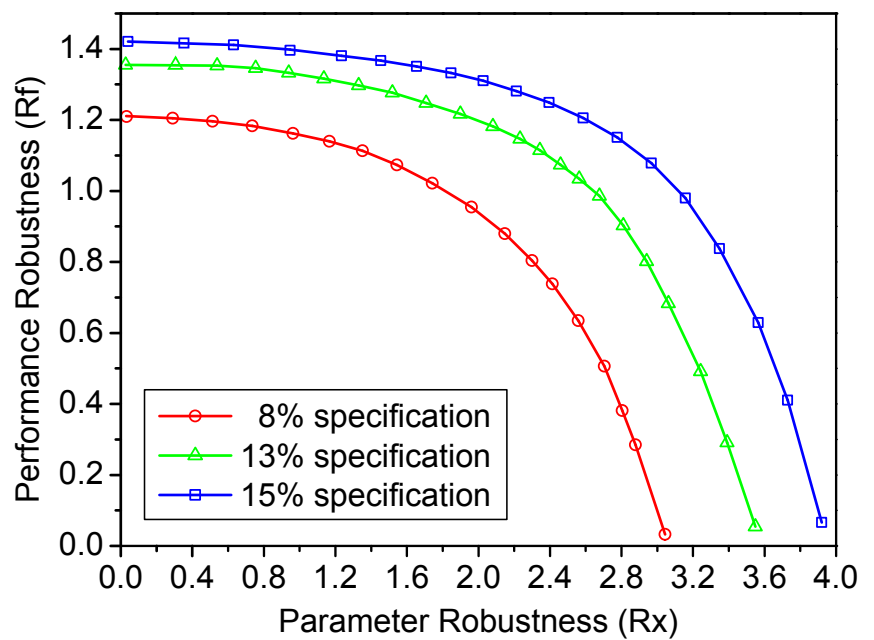

Figure 7: Robustness Pareto surfaces with different performance specification values

posed method provides the designer with the flexibility to choose the most appropriate solution(s) according to specific design requirements. In addition, the runtime of the two-way mapping procedure without using surrogate modeling is also presented to demonstrate the efficiency by employing surrogate modeling when solving the robustness optimization problem. At different performance specifications, an up to $124 \mathrm{x}$ speedup has been achieved by the proposed surrogate-based approach.

Table 3: Runtime comparisons among various methods at different yield specifications

\begin{tabular}{|c|c|c|c|c|}
\hline $\begin{array}{c}\text { Performance } \\
\text { Specs. }\end{array}$ & Two-way Mapping & $\begin{array}{c}\text { Forward } \\
\text { Mapping }\end{array}$ & $\begin{array}{c}\text { Backward } \\
\text { Mapping }\end{array}$ \\
\hline $8 \%$ & $25.39 \mathrm{~s}$ & $3145 \mathrm{~s}$ & $1.97 \mathrm{~s}$ & $0.48 \mathrm{~s}$ \\
\hline $10 \%$ & $26.88 \mathrm{~s}$ & $3148 \mathrm{~s}$ & $1.90 \mathrm{~s}$ & $0.71 \mathrm{~s}$ \\
\hline $13 \%$ & $35.22 \mathrm{~s}$ & $3158 \mathrm{~s}$ & $2.15 \mathrm{~s}$ & $0.63 \mathrm{~s}$ \\
\hline $15 \%$ & $42.41 \mathrm{~s}$ & $3163 \mathrm{~s}$ & $2.41 \mathrm{~s}$ & $0.58 \mathrm{~s}$ \\
\hline
\end{tabular}

\section{Conclusions}

This paper proposed a new robust design framework considering both parameter robustness and performance robustness. For any feasible design candidate, its robustness metrics can be quantified by employing the ElasticR model. A surrogate-based optimization procedure are then presented to explore a set of optimal nominal designs that achieves maximum robustness as well as minimum performance fluctuation. The efficiency of the proposed methodology was demonstrated on an opamp circuit using 90nm technology.

\section{Acknowledgements}

This work was supported by the National Science Foundation under grant nos. IIA-0926278, CCF-0915537 and
CCF-0832282. The authors would like to thank Mr. Kiran Potluri for helping design the operational amplifier circuit.

\section{References}

[1] P. G. Drennan, C. McAndrew, Understanding mosfet mismatch for analog design, IEEE Journal of Solid-State Circuits 38 (3) (2003) 450-456.

[2] P. G. Drennan, M. L. Kniffin, Implications of proximity effects for analog design, in: Proceedings of the Custom Integrated Circuits Conference(CICC), 2006, pp. 169-176.

[3] K. Shepard, D. Maynard, Variability and yield improvementrules, models, and characterization, in: Proceedings of the International Conference on Computer-Aided Design (ICCAD), 2006, pp. 834-835.

[4] C. Visweswariah, K. Ravindran, K. Kalafala, S. G. Walker, S. Narayan, First-order incremental block-based statistical timing analysis, in: Proceedings of the Design Automation Conference (DAC), 2004, pp. 331-336.

[5] R. R. Rao, A. Devgan, D. Blaauw, D. Sylvester, Parametric yield estimation considering leakage variability, in: Proceedings of the Design Automation Conference (DAC), 2004, pp. 442447.

[6] A. Srivastava, K. Chopra, S. Shah, D. Sylvester, D. Blaauw, A novel approach to perform gate-level yield analysis and optimization considering correlated variations in power and performance, IEEE Transaction on Computer-Aided Design of Integrated Circuits and Systems 27 (2) (2008) 272-285.

[7] A. Ben-Tal, A. Nemirovski, Robust convex optimization, Mathematics of Operations Research 23 (4) (1998) 769-805.

[8] D. Bertsimas, D. B. Brown, C. Caramanis, Theory and applications of robust optimization, SIAM Review 53 (3) (2011) 464-501

[9] S.-K. Kim, Enhanced evaluation method of sigma level in sixsigma activities, in: Proceedings of the International Conference on Management of Innovation and Technology (ICMIT), 2008, pp. 1116-1119.

[10] M. Nataraj, V. P. Arunachalam, K. G. Suresh, Optimising planer cam mechanism in printing machine for quality improvement using taguchi method: risk analysis with concurrent engineering approach, International Journal of Computer Applications in Technology 26 (3) (2006) 164-173.

[11] R. K. Roy, A Primer on the Taguchi method, Van Nostrand Reinhold, New York, NY, 1990.

[12] G. Taguchi, Quality engineering (taguchi methods) for the development of electronic circuit technology, IEEE Journal of Solid-State Circuits 44 (2) (1995) 225-229.

[13] D. H. Stamatis, Six Sigma Fundamentals: A Complete Guide to the System, Methods, and Tools, Productivity Press, New York, NY, 2004.

[14] S. K. Tiwary, P. K. Tiwary, R. A. Rutenbar, Generation of yield-aware pareto surfaces for hierarchical circuit design space exploration, in: Proceedings of the Design Automation Conference (DAC), 2008, pp. 1116-1119.

[15] K. Heloue, C. Kashyap, F. Najm, Quantifying robustness metrics in parameterized static timing analysis, in: Proceedings of the International Conference on Computer-Aided Design (ICCAD), 2009, pp. 209-216.

[16] J. Singh, V. Nookala, Z. Luo, S. Sapatnekar, A geometric programming-based worst case gate sizing method incorporating spatial correlation, IEEE Transactions on Computer-Aided Design of Integrated Circuits and Systems 53 (3) (2011) 464501.

[17] Y. Xu, K.-L. Hsiung, X. Li, L. T. Pileggi, S. P. Boyd, Regular analog/RF integrated circuits design using optimization with recourse including ellipsoidal uncertainty, IEEE Transactions on Computer-Aided Design of Integrated Circuits and Systems 28 (5) (2009) 623-637.

[18] J. Sun, P. Gupta, J. Roveda, A new uncertainty budgeting based method for robust analog/mixed-signal design, in: Proceedings 
of the Design Automation Conference (DAC), 2012, pp. 529535.

[19] H. You, M. Yang, D. Wang, X. Jia, Kriging model combined with latin hypercube sampling for surrogate modeling of analog integrated circuit performance, in: Proceedings of the International Symposium on Quality Electronic Design (ISQED), 2009, pp. $554-558$.

[20] F. Yengui, L. Labrak, P. Russo, F. Frantz, N. Abouchi, Optimization based on surrogate modeling for analog integrated circuits, in: Proceedings of the International Conference on Electronics, Circuits \& Systems (ICECS), 2012, pp. 9-12.

[21] M. B. Yelten, P. D. Franzon, M. B. Steer, Surrogate modelbased analysis of analog circuitspart i: Variability analysis, IEEE Transactions on Devices and Materials Reliability 11 (3) (2011) 458-465.

[22] S. Gunawan, S. Azarm, Non-gradient based parameter sensitivity estimation for single objective robust design optimization, Journal of Mechanical Design 126 (3) (2004) 395-402.

[23] S. Gunawan, S. Azarm, Multi-objective robust optimization using a sensitivity region concept, Structural and Multidisciplinary Optimization 29 (1) (2005) 50-60.

[24] S. Gunawan, S. Azarm, A feasibility robust optimization method using a sensitivity region concept, Journal of Mechanical Design 127 (5) (2005) 858-868.

[25] M. Li, S. Azarm, A. Boyars, A new deterministic approach using sensitivity region measures for multi-objective and feasibility robust design optimization, Journal of Mechanical Design 128 (4) (2006) 874-883.

[26] M. Li, G. Li, S. Azarm, A kriging metamodel assisted multiobjective genetic algorithm for design optimization, Journal of Mechanical Design 130 (3) (2008) 1-10.

[27] C. Audet, A. J. Booker, J. E. D. Jr., P. D. Frank, D. Moore, A surrogate-model-based method for constrained optimization, in: Proceedings of the Symposium on Multidisciplinary Analysis and Optimization, 2000, p. 4891.

[28] A. J. Booker, J. E. D. Jr., P. D. Frank, D. B. Serafini, V. Torczon, M. W. Trosset, A rigorous framework for optimization of expensive functions by surrogates, Structural Optimization 17 (1) (1999) 1-13.

[29] M. Lobo, L. Vandenberghe, S. Boyd, H. Lebret, Applications of second-order cone programming, Linear Algebra and its Applications $284(1-3)(1998)$ 193-228.

[30] I. Y. Kim, O. L. de Weck, Adaptive weighted sum method for bi-objective optimization: Pareto front generation, Structural and Multidisciplinary Optimization 29 (2) (2005) 149-158.

[31] J. Koski, Defectiveness of weighting method in multicriterion optimization of structures, Communications in Applied Numerical Methods 1 (6) (2005) 333-337.

[32] I. Das, J. E. Dennis, Normal-boundary intersection: a new method for generating pareto optimal ooints in multicriteria optimization problems, SIAM Journal on Optimization 8 (3) (1998) 631-657.

[33] M. H. Bakr, J. W. Bandler, K. Madsen, J. Sondergaard, An introduction to the space mapping technique, Optimization and Engineering 2 (4) (2001) 369-384.

[34] J. W. Bandler, K. Madsen, Surrogate modelling and space mapping for engineering optimization, Optimization and Engineering 2 (4) (2001) 367-368.

[35] C. Audet, J. E. D. Jr., Pattern search algorithms for mixed variable programming, SIAM Journal of Optimization 11 (3) (2000) 573-594. 
Dear Editor-in-Chief, Associate Editor and Reviewers,

Thank you for taking your time to provide us your comments and suggestions that helped us improve the quality of this paper. We have revised the paper to incorporate all reviewers' suggestions (the newly added contents are clearly marked in red color in this revised manuscript). Please see below the specific responses to individual comments.

Sincerely

Jin Sun et al.

$* * * * * * * * * * * * * *$

Reviewer\#1:

The paper brings together several concepts from different fields to form a consistent whole. Initially, the L-norm and the ElasticR method are introduced as a measure of robustness quantification. Adapted from different fields, these measures are interesting. Then, the difference between parameter and performance robustness are provided. Again, the same measures are applied to performance robustness. Then, an optimization is run for $\mathrm{Rx}$ against Rf. The AWS approach is effective, but not the most effective approach to find the Pareto front. Surrogate models are used to ease the optimization procedure.

Is this really the most efficient way of obtaining a $2 \mathrm{D}$ Pareto front? There are many other algorithms which create fronts of implicit functions. Please comment on why you have chosen surrogate modeling.

ANS: We thank the reviewer for raising this question. There are a number of other algorithms that deal with implicit functions by using approximation or fitting techniques. First, these nonsurrogate-based approaches generally may lead to more expensive functions (compared with surrogate modeling method), while the SMF method employed in this work provides a sequence of costeffective approximations to performance functions. Second, fitting-based methods require large number of sampling points and may cause serious over-fitting issues. The polling procedure in SMF method guarantees that the surrogate model converges to the true performance function without over-fitting concerns. Third, SMF method does not require any assumption of surrogates and therefore eases the tractability in solving the design for robustness problem. The clarification regarding this question has been made in the first paragraph of Section 3.2, right column on Page 5.

We also would like to clarify the reason why we have chosen AWS method for generating the Pareto front. Though there are various algorithms for solving multi-objective problems (e.g. conventional weighted sum method and normal boundary insertion method), the advantage of AWS method is its ability to provide a well-distributed Pareto front even in non-convex regions. A clarification has been made in the first paragraph of Section 3.1, left column on Page 5.

How long does your optimization take on your $2.5 \mathrm{GHz}$ quad core machine?

ANS: Thank you for this valid point. In this revised manuscript we have included a runtime comparison between using the proposed two-way mapping method (mutli-objective procedure) and oneway backward/forward mapping method (singleobjective procedure). At different performance specification values, the CPU time by using different methods are listed.

Please refer to Table 3 and its related discussion (the last paragraph, right column on Page 8) for the runtime statistics. Please kindly note that all experiments have been re-evaluated on a new 3.4GHz quad-core machine with a 16-GB memory.

Also, please explain the practical significance of Figure 6. How can a designer use this front in his/her design?

ANS: This figure was presented to demonstrate the accuracy of the robustness optimization results: the generated Pareto surface is on the boundary of $R_{x}-R_{f}$ space consisting of Monte-Carlo sampling results. Since each sample of feasible design candidate corresponds to a pair of robustness metrics $\left(R_{x}, R_{f}\right)$ in robustness space, the complete robustness space (formed by all feasible design candidates) can be approximated by Monte-Carlo results. According to the definition of Pareto optimality, all Pareto solutions are supposed to be distributed on the boundary of robustness space. Depending on specific robustness requirements, the designer may choose a specific solution on the generated Pareto surface as the nominal design that yields the Pareto-optimal robustness metric. Above explanations have been included in the first paragraph, left column on Page 8 .

Presumably, on could build a sensitivity matrix around the nominal design point and perturb each parameter, checking the performance deviation. By simplifying this matrix, it would be possible to extract the most important parameter/performance pairs and combine them linearly, obtaining a similar plot. Depending on the number of parameters, this could be much faster. It would also give information about the significance of the parameters. Why would your approach be more successful/desirable than such a simple minded sensitivity matrix?

ANS: Thank you for bringing up this concern to our attention. We agree that sensitivity matrix is a well-studied approach. It makes sense to use sensitivity to identify the significance of design parameters to circuit performance. However, sen- 
sitivity would not be able to reveal robustness of the design. In some cases, high sensitivity may lead to low robustness (due to the large spread in performance metric and there the low parametric yield). We have discussed and clarified the concept of robustness in the discussion of feasible region, and the definition of robustness (refer to Section 2.1). The significance of design parameter is thus discussed under the scope of robustness, not sensitivity.

ANS: More importantly, the proposed approach not only quantifies/optimizes robustness of design parameters, but also the robustness of performance fluctuation propagated from parameter variations. We think that a co-optimization of parameter robustness and performance robustness leads to more robust circuit designs.

Finally, there are occasional language mistakes. Please re-read the paper.

ANS: Thank you for your suggestion. We have done a thorough proofreading and attempted to correct those grammatical mistakes.

\section{$* * * * * * * * * * * * * *$}

Reviewer\#2:

The paper addresses a relevant topic to this journal which is analog IC design automation.

The proposed approach, surrogate approach, must be put into context knowing that, nowadays, there are various solutions published in major EDA conferences (DATE, DAC, ICCAD) and journals (IEEE TCAD, ACM TODAES and Integration, The VLSI Journal) applying optimization-based approaches without the need of surrogate models to address successfully analog IC design (including robust design and even layout-aware solutions).

(1) The authors should review the introduction part including references to those works or creating a new section "Analog IC Design Automation: Overview".

ANS: Thank you for your valuable suggestion. We have revised the Introduction part and have included the discussion of optimization-based approaches without surrogate modeling. The newly added references and their related discussions can be referred to the second paragraph, right column on Page 1.

We also would like to clarify that, while these optimization tools can provide a set of potential solutions, we think the surrogate idea is still valid as it can perform as a higher layer to these tools to help automatically sort out the best solutions among all sets. Also, most existing tools tend to use continuous models for optimization, which is not required for the surrogating approach used in this work (SMF method). The polling procedure in the SMF method guarantees that the surrogate model converges to the performance function without any assumptions of surrogates and therefore facilitates the solver when solving the robust design problem. This clarification can be found in the first paragraph of Section 3.2, right column on Page 5.

(2) Even in term of surrogate model approaches, in the area of analog IC design, more recent references can be found in literature.

ANS: We have also included a new paragraph discussing recent references on surrogate modeling in analog circuit design. Please refer to the second paragraph, left column on Page 2 in Introduction part.

Considering the proposed approach, it is well described in sections 2 and 3. (1) One point that is avoided but should be discussed is the reuse or not of the surrogate model.

ANS: Thank you for this valid suggestion. The reuse of the surrogate model or not depends on whether the designer is satisfied with the accuracy of surrogate model. The SMF method used in this work relies on a polling procedure to converge to the performance function. The surrogate model can be reused if additional points are selected for surrogating, and the polling procedure thus will continue. This discussion has been added in the second paragraph from the bottom, left column on Page 6.

(2) Another point that should be explained is how do you guarantee that the point in the Pareto front shown is of interest for the designer, i.e., can't we find a Pareto Front of solutions for any point in the robust metrics Pareto Front shown in Fig. 6?

ANS: This figure was presented to demonstrate that the generated Pareto surface is on the boundary of $R_{x}-R_{f}$ space consisting of Monte-Carlo sampling results. The AWS algorithm guarantees that all Pareto solutions are evenly distributed on the boundary of robustness space. Therefore, depending on specific robustness requirements, the designer may choose a specific solution on the generated Pareto surface as the nominal design that leads to the robustness metric in Pareto-optimal sense. More explanations can be found in the first paragraph, left column on Page 8.

(3) Considering the relevance of layout issues like electromigration and parasitics during the sizing, how would you take this into account in order to compete with other existing solutions?

ANS: Thank you for raising this question. We think that the proposed method can be easily extended to incorporate the layout relevance as a 
model that affects sizing, i.e. we can incorporate the relevance of layout issues as an additional constraint function in the formulated optimization model.

In terms of results, the authors should include: (1) Results of optimization with and without surrogate model.

ANS: We appreciate the reviewer for raising this important issue. We have realized that a comparison of results with and without surrogate modeling is necessary to strengthen this work. A new table conducting such comparison as well as a new paragraph discussing the results have been included in this revised manuscript. Please refer to Table 1 and the second paragraph, left column on Page 8 for the details. A minor difference in the results by two methods can be observed.

(2) Describing the computational overhead for building the surrogate model.

ANS: In this revised manuscript we have presented the runtime statistics for all methods used in experiments. At different performance specification values, the CPU time of two-way mapping method using surrogate modeling is listed in the second column of Table 3 .

(3) The Pareto front for 2 performance objectives, for example, GBW, PM, Gain, etc.

ANS: We clarify that the two objectives in the generated Pareto front are parameter robustness and performance robustness, the two robustness metrics defined in (6) and (8). Also, the robustness metrics are evaluated under three performance constraints, gain-bandwidth, phase margin, and slew rate. Though for illustrative purpose, all the figures in Section 2 use two performance functions to interpret the concepts, the robustness metrics developed in this work can be applied to scenarios with multiple performance constraints. This clarification has been added in the second paragraph, left column on Page 5.

(4) Consider examples which allow the comparison with other approaches.

ANS: In this revised manuscript we have added Table 1 to present the comparison of robustness metrics with surrogate modeling and without surrogate modeling, as well as Table 3 to present the comparison of CPU time among different approaches. Also, Table 2 provides the comparison of robustness metrics by using two-way mapping method and one-way mapping methods. Please refer to Table 1, Table 2, Table 3 as well as their related text for the comparison results.

\section{Reviewer\#3:}

This paper presents a design framework to obtain a Pareto surface of robustness metrics (parameter robustness and performance robustness). The proposed methodology includes forward and backward mapping procedures, an ElasticR method to quantify the value of both robustness metrics and a surrogate-based robust design framework; finally some results are presented.

However, I have a lot of concerns about this work: ${ }^{*}$ In section 2, both robustness metrics are well explained but the trade-off between them is not clear; for instance, why better parameters robustness would lead to worse performance robustness?

ANS: We thank the reviewer for raising this question. We would like to clarify that better parameter robustness does not necessarily lead to worse performance robustness. These two robustness metrics are incorporated in a multiobjective optimization framework, and therefore are optimized simultaneously. The results obtained by this multi-optimization framework are design solutions that provides Pareto-optimal robustness metrics. Use one Pareto solution in Figure 6, $\left(R_{x}=2.2542, R_{f}=0.9435\right)$, for example, it is called Pareto-optimal because there exists no other solutions such that $R_{x}$ has a better value than 2.2542, while $R_{f}$ value is the same or better than 0.9435. Sorry for causing this confusion.

* It is not clear at all when the authors are talking about process variability (e.g., tox variations) or changes in the nominal design (e.g., changing the width of the transistors). One would expect that the parameter robustness is primarily defined by the nominal design and the authors often take a process parameter (said tox) as a parameter that the designer can tweak to attain better performances, which is not true.

ANS: Sorry again about this confusion. To be accurate, the parameter robustness in this work is evaluated for each nominal design (that is feasible) considering the process variations around the nominal values. Each feasible nominal design corresponds to a robustness metric evaluated in parameter space (refer to (6)) as well as a robustness metric evaluated in performance space (refer to (8)). By the co-optimization of these two robustness metrics, the proposed two-way mapping procedure aims at exploring nominal design solutions with Pareto-optimality of parameter robustness and performance robustness.

* In section 3, the use of surrogate-based optimization is explained, but the general flow of this methodology is not clear: how are backward mapping and forward mapping done? When is a circuit simulation needed in this flow? 
ANS: The backward mapping is used to determine the mapping relationship between parameter robustness and design parameters, while the forward mapping is used to determine the mapping relationship between performance robustness and design parameters. The backward mapping and forward mapping are done whenever the multi-optimization framework needs to evaluate the objective function (simply a weighted sum of the two robustness metrics). Please kindly note that the robustness metrics are determined by solving sub-optimization procedures (6) and (8), and therefore are implicit functions in terms of design parameters. In this situation, the surrogate modeling would be necessary to address this issue. Circuit simulations are consequently required to build surrogates for determining robustness metrics defined in (6) and (8). This clarification has been made in the first paragraph, right column on Page 5, right before Section 3.2,

* The result of this flow is a Pareto surface, but what is the starting point for a designer? They just define the performance specifications or is anything more needed (i.e. a nominal design, or a variation range for each sizing parameter)? Again, it is not clear if the flow deals with just a single design or with a region or group of nominal designs.

ANS: We would like to clarify that this flow deals with a region of nominal designs rather than a single nominal design. As we explained in previous Q\&A, each feasible nominal design corresponds to a pair of parameter robustness performance robustness, and the proposed co-optimization flow aims at exploring nominal design solutions that yield Pareto-optimality robustness metrics.

The Pareto surface generated by this flow in fact is a set of Pareto-optimal solutions corresponding different weighting factors in (11). With these evenly-distributed Pareto solutions, the designer may decide a specific design criteria and choose an appropriate weighting factor $\alpha$ as the starting point. For example, if the design expects similar level of robustness in parameter space and performance space, he/she may choose $\alpha=0.5$ so both robustness metrics are equivalently weighted.

* The experimental results do not prove enough the usefulness of the proposed methodology, and more importantly, how the generated Pareto surface can help the designer.

ANS: We thank the reviewer for bringing up this concern to our attention. As we explained previously, the Pareto surface is a set of Pareto solutions, and each Pareto solution corresponds to a particular weighting factor in the multi-optimization framework. A complete and well-distributed Pareto surface provides the designer with the flexibility to choose the most ap- propriate solution(s) according to specific design requirements. For example, if a better robustness metric is expected in parameter/performance space, a higher weight should be assigned the parameter/performance robustness metric. More discussion on this issue has been included in the first paragraph, left column on Page 8.

* In addition, the experimental result is compared with a Monte-Carlo simulation using 2000 samples. However, no information about the required CPU time is shown. What's the gain from using the proposed methodology when compared to simple Monte-Carlo analysis?

ANS: Thank you for this useful comment. In the newly added Table 3 we have provided the runtime statistics for all methods used in experiments. The two-way mapping procedure without surrogate modeling relies on the Monte-Carlo sampling data to obtain Pareto-optimal robustness solutions. At different performance specifications, an up to $124 \mathrm{x}$ speedup has been achieved by the proposed surrogate-based approach.

In summary, the general flow of the proposed methodology needs a more thorough explanation; also the experimental results have to show more clearly how the designer would use the information from this Pareto-surface.

Check also the whole paper for typos and grammar mistakes.

ANS: Thank you for pointing out this problem. We have proofread the whole paper and attempted to eliminate these mistakes. 